\title{
Assessment of coronary artery disease risk in 5463 patients undergoing cardiac surgery: When is preoperative coronary angiography necessary?
}

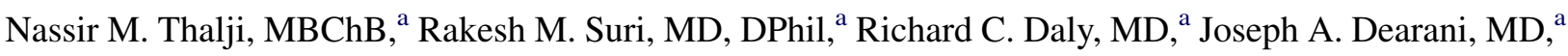 \\ Harold M. Burkhart, MD, ${ }^{a}$ Soon J. Park, MD, ${ }^{a}$ Kevin L. Greason, MD, ${ }^{a}$ Lyle D. Joyce, MD, ${ }^{a}$ \\ John M. Stulak, MD, ${ }^{\mathrm{a}}$ Marianne Huebner, PhD, ${ }^{\mathrm{b}}$ Zhuo Li, MSc, ${ }^{\mathrm{b}}$ Robert L. Frye, $\mathrm{MD},{ }^{\mathrm{c}}$ and \\ Hartzell V. Schaff, MD ${ }^{\mathrm{a}}$
}

Objectives: We sought to critically analyze the routine use of conventional coronary angiography (CCA) before noncoronary cardiac surgery and to assess clinical prediction models that might allow more selective use of CCA in this setting.

Methods: We studied 5463 patients undergoing aortic valve surgery, mitral valve surgery, or septal myectomy with or without coronary artery bypass grafting from 2001 to 2010. Preoperative CCAs were evaluated for the presence of significant coronary artery disease (CAD). Random forests and logistic regression methods were used to determine the predictors of significant $(\geq 50 \%)$ coronary stenosis.

Results: Preoperative CCA was performed in 4711 patients (86\%). Two thirds of those with angina, previous myocardial infarction, or percutaneous coronary intervention had significant CAD found on CCA, versus one third of patients free of these risk factors $(P<.001)$. Among 3019 patients without angina, previous myocardial infarction or percutaneous coronary intervention, older age, male gender, diabetes, and peripheral vascular disease independently predicted significant CAD $(P<.001$ for all; C-index $=0.74)$. Specifically, a multivariate model with these variables identified $10 \%$ (301 of 3019) of patients as having a low $(\leq 10 \%)$ probability of coronary stenosis, of whom fewer than $5 \%$ had significant CAD and fewer than $1 \%$ had left main or triple-vessel coronary disease.

Conclusions: In the absence of angina, previous myocardial infarction, or percutaneous coronary intervention, preoperative CCA identified significant CAD in only one third of patients. Our clinical prediction models could enhance the identification of patients at low risk of significant CAD for whom CCA might potentially be avoided before cardiac surgery. This strategy may improve the efficiency of cardiac surgical care delivery by diminishing procedure-related morbidity and offering significant cost savings. (J Thorac Cardiovasc Surg 2013;146:1055-64)

Supplemental material is available online.

\footnotetext{
From the Division of Cardiovascular Surgery, ${ }^{a}$ Department of Biomedical Statistics and Informatics, ${ }^{\mathrm{b}}$ and Division of Cardiovascular Diseases, ${ }^{\mathrm{c}}$ Mayo Clinic College of Medicine, Rochester, Minn.

The Division of Cardiovascular Surgery, Mayo Clinic College of Medicine, funded the present study, including the statistical analysis.

Disclosures: The Division of Cardiovascular Surgery has a research grant titled "Randomized biological aortic valve replacement" funded equally by Edwards LifeSciences, St. Jude Medical, and the Sorin Group. Dr Suri is the Principal Investigator for the PERCEVAL IDE trial funded by the Sorin Group. Dr Park serves on the advisory board for Thoratec and the Cleveland Clinic. None of the disclosures pertain to the present investigation. All other authors have nothing to disclose with regard to commercial support.

Read at the 93rd Annual Meeting of The American Association for Thoracic Surgery, Minneapolis, Minnesota, May 4-8, 2013.

Received for publication May 5, 2013; revisions received June 10, 2013; accepted for publication June 26, 2013; available ahead of print Sept 6, 2013.

Address for reprints: Rakesh M. Suri, MD, DPhil, Division of Cardiovascular Surgery, Mayo Clinic College of Medicine, 200 First St, SW, Rochester, MN 55905 (E-mail: suri.rakesh@mayo.edu).

$0022-5223 / \$ 36.00$

Copyright (c) 2013 by The American Association for Thoracic Surgery

http://dx.doi.org/10.1016/j.jtcvs.2013.06.046
}

Earn CME credits at

http://cme.ctsnetjournals.org
In patients presenting for noncoronary cardiac surgery, underlying coronary obstruction, if severe and not bypassed, can confer deleterious effects on perioperative ${ }^{1}$ and longterm outcomes. ${ }^{2}$ Thus, the identification of coexistent coronary artery disease (CAD) using conventional coronary angiography (CCA) has long been paramount in the assessment of cardiac surgical candidates. ${ }^{3}$ However, owing to the broad guidelines outlining which patients undergoing cardiac surgery warrant angiography, ${ }^{4}$ the practice of screening CCA has become ubiquitous. Although highly sensitive in identifying patients with $\mathrm{CAD}$, it has been proposed that guideline specificity might be as low as $1 \%$ to $3 \%{ }^{5,6}$ When considered alongside recent trials demonstrating no advantage for revascularization compared with medical therapy in reducing the risk of myocardial infarction (MI) or death-except in those with the most extensive coronary disease ${ }^{7,8}$ - the suggestion has been that a 


$$
\begin{aligned}
& \text { Abbreviations and Acronyms } \\
& \begin{aligned}
\text { AVS } & =\text { aortic valve surgery } \\
\text { CAD } & =\text { coronary artery disease } \\
\text { CCA } & =\text { conventional coronary angiography } \\
\text { CABG } & =\text { coronary artery bypass grafting } \\
\text { MI } & =\text { myocardial infarction } \\
\text { MVS } & =\text { mitral valve surgery } \\
\text { OR } & =\text { odds ratio } \\
\text { PCI } & =\text { percutaneous coronary intervention } \\
\text { PVD } & =\text { peripheral vascular disease } \\
\text { SM } & =\text { septal myectomy }
\end{aligned}
\end{aligned}
$$

disproportionate number of patients coming to cardiac surgery might be unnecessarily subjected to an invasive and costly procedure.

Although the incidence of significant, but asymptomatic, $\mathrm{CAD}$ in the general population remains unclear, a recent report from the National Cardiovascular Data Registry detailed that only one third of approximately 400,000 patients undergoing elective CCA were found to have significant $\mathrm{CAD}{ }^{9}$ However, patients undergoing valvular heart surgery - in whom coronary disease burden may be increased ${ }^{10}$-were excluded from their analysis. Consequently, the true diagnostic yield of preoperative CCA in patients presenting for noncoronary cardiac surgery remains unclear, and the need persists for an updated risk stratification tool, allowing clinicians to consistently assess the probability of concomitant CAD. We therefore performed a large-scale retrospective study of patients undergoing noncoronary cardiac surgery, with the aims of (1) delineating the burden of underlying obstructive CAD, and (2) developing simple bedside models to predict the likelihood of significant coexistent coronary stenosis.

\section{METHODS \\ Study Design and Population}

We performed an analysis of patients aged 18 years or older who had undergone aortic valve surgery (AVS), mitral valve surgery (MVS), or septal myectomy (SM), with or without concomitant coronary artery bypass grafting (CABG), from 2001 to 2010 . The exclusion criteria were previous sternotomy, concomitant major procedures other than $\mathrm{CABG}$ (eg, thoracic, aortic, or tricuspid valve surgery), active endocarditis, and ischemic valve disease. The Mayo Clinic institutional review board approved the present investigation.

Subjects were identified from the Division of Cardiovascular Surgery's patient database. A total of 10,677 consecutive patients had undergone AVS, MVS, and/or SM at the Mayo Clinic (Rochester, Minn) from January 1, 2001 to December 31, 2010, of whom 5463 met the enrollment criteria (Figure 1). The patients were divided into 2 groups according to the use of CCA within 6 months of surgery ( $n=4711$ CCA vs $n=752$ no CCA).

We further categorized the patients undergoing CCA into (1) those with baseline angina symptoms, previous $\mathrm{MI}$, or previous percutaneous coronary intervention (PCI) $(\mathrm{n}=1692)$ and (2) those patients free of these risk factors $(\mathrm{n}=3019)$. The latter cohort was used to derive models predicting the likelihood of significant CAD at cardiac surgery. In an effort to maximize the predictive accuracy, we constructed separate models for patients who had undergone AVS ( $\mathrm{n}=1668)$, MVS $(\mathrm{n}=1070)$, or SM $(\mathrm{n}=281)$. Patients undergoing multiple procedures were analyzed in 1 category only according to a priori rules. First, given the similarity in risk factors and/or pathophysiologic mechanisms underlying the development of CAD and aortic valve disease, ${ }^{11-13}$ patients undergoing AVS were analyzed in this category, irrespective of concomitant procedures. Second, patients undergoing combined MVS plus SM were analyzed according to the primary surgical indication, as documented by the treating physician.

\section{Clinical Data}

The Division of Cardiovascular Surgery's database and the medical records were reviewed for patient demographics, medical history, baseline symptoms, and perioperative outcomes. The variables evaluated when constructing the prediction models included age, gender, diabetes mellitus, peripheral vascular disease (PVD), hypertension, hypercholesterolemia, smoking history, family history of CAD, cerebrovascular accident, New York Heart Association functional class III-IV, impaired ejection fraction $(<50 \%)$, and obesity (body mass index $\left.\geq 30 \mathrm{~kg} / \mathrm{m}^{2}\right)$. These variables were defined in keeping with the standard criteria set forth by the Society of Thoracic Surgeons as a part of the National Adult Cardiac Surgery Database. $^{14}$

The primary endpoint was significant $\mathrm{CAD}$, defined as angiographic evidence of $50 \%$ or more luminal stenosis of any of the epicardial coronary vessels, including side branches. ${ }^{15}$ The secondary endpoints were high-risk coronary disease, defined as $50 \%$ or more stenosis of the left main coronary artery or all 3 major epicardial vessels, and the need for CABG at baseline surgery.

\section{Coronary Angiograms}

The results of the CCAs performed within 6 months of surgery were available from the Division of Cardiovascular Disease Coronary Catheterization database and by review of the medical records from our institution and outside centers. CAD burden was assessed by the percentage of luminal stenosis of coronary vessels as documented at the time of study.

\section{Statistical Analysis}

Categorical variables are summarized as counts and percentages and were compared between groups using the chi-square test. Continuous variables were compared using $t$ tests or the Wilcoxon rank-sum test, as appropriate.

Univariate logistic regression models identified the risk factors for CAD. A multivariate model for the overall cohort was constructed using random forests for variable selection, and odds ratios were estimated by entering these variables into logistic regression models. Random forests are a nonparametric ensemble partitioning classification method that calculates measures of prediction accuracy and variable importance. ${ }^{16}$ By resampling subjects and predictor variables, it can account for nonlinear effects for variables and multi-way interactions between variables. The contribution of each covariate to the overall prediction accuracy of CAD was evaluated by measuring the changes in model accuracy when the factor was not considered. A feature selection algorithm is preferable to stepwise selection algorithms because the latter overestimate effect sizes and underestimate standard errors. ${ }^{17}$ Variables identified using this method according to prediction accuracy and variable importance were then entered into multivariate logistic regression models to predict CAD.

To enhance utility by physicians at the bedside, multivariate models limited to 4 main effects were selected according to the concordance index in all subsets regression. Such models were constructed for the overall cohort and separately for AVS, MVS, and SM subgroups. Internal cross-validation with 200 bootstrap samples was used. The predicted probabilities of $\mathrm{CAD}$ were calculated. After consultation with expert 


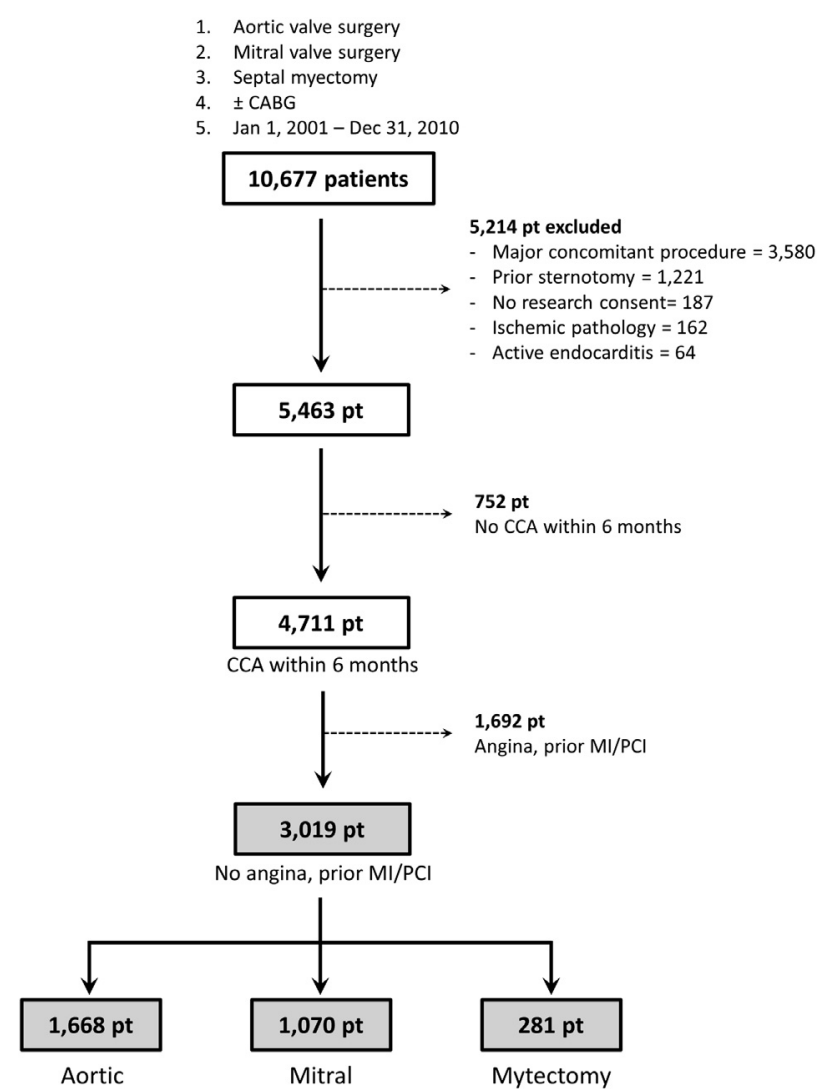

FIGURE 1. Identification of study subjects. $C A B G$, Coronary artery bypass grafting; $C C A$, conventional coronary angiography; $M I$, myocardial infarction; $P C I$, percutaneous coronary intervention.

cardiologists and cardiovascular surgeons, a low probability for significant CAD was defined as $10 \%$ or less. This cutpoint has been used in multiple cardiovascular studies ${ }^{18-21}$ and has previously been shown to be a valid threshold for the classification of "low risk." 22

The analyses were performed using Statistical Analysis Systems, version 9.13, software (SAS Institute, Cary, NC), and the R package randomForest, version 4.6-7, in the statistical software package, $\mathrm{R}$, version $2.15 .3^{23}$

\section{RESULTS}

\section{Baseline Clinical Characteristics}

The baseline characteristics are listed in Table 1, stratified by whether or not patients underwent preoperative CCA within 6 months of surgery. Mean patient age was $65 \pm 15$ years, and 3426 patients were men $(63 \%)$. Preoperative CCA was performed in 4711 patients $(86 \%)$. As expected, those who did not undergo CCA were younger and less symptomatic at index surgery. Coincident with fewer baseline comorbidities, patients without preoperative CCA also experienced fewer perioperative complications, including lower operative mortality.

\section{CAD Burden}

Of the 4711 patients with preoperative CCA, 2139 (45\%) had significant $\mathrm{CAD}$, one third of whom $(\mathrm{n}=744)$ had high-risk (see "Methods") CAD. Baseline CABG was performed in only 1706 of the 4711 patients $(36 \%)$ evaluated by CCA.

\section{Patients With Angina, Previous MI, and Previous PCI}

Patients with angina, previous MI, or previous PCI constituted $36 \%$ of the population (1692 of 4711) undergoing CCA. In the presence of these established risk factors, the age- and gender-adjusted odds of significant CAD was 4-fold greater than in the absence of these 3 variables (odds ratio [OR], 3.94; $95 \%$ confidence interval, 3.44-4.53; $P<.001$ ). Two thirds of the patients (1125 of 1692) with angina, previous MI, or PCI had significant CAD on CCA versus one third of patients (1014 of 3019) free of these risk factors. Baseline CABG was thus performed in 958 of 1692 patients $(57 \%)$ with angina, previous MI, or PCI and in only 748 of 3019 of patients $(25 \%)$ without these features on preoperative history. Because of the preponderance of significant CAD among the patients with these major risk factors, and given our objective of identifying those with a low disease probability, we focused on the 3019 patients without angina, previous MI, or PCI when constructing prediction models.

\section{CAD Prediction Among All Patients Without Angina, Previous MI, or PCI}

The univariate predictors of significant CAD in the 3019 patients without angina or previous MI or PCI are outlined in Table E1. Multivariate predictors of CAD included older age, male gender, diabetes, PVD, family history of CAD, hypertension, hypercholesterolemia, and smoking history $(\mathrm{C}$-index $=0.76$; Table E1).

The 4-variable model consisting of older age (OR, 1.4 per 5 years), male gender (OR, 1.9), diabetes (OR, 2.3), and PVD (OR, 2.7) had the best discriminative power for predicting $\mathrm{CAD}(P<.001$ for all; $\mathrm{C}$-index $=0.74)$. According to this model, $10 \%$ (301 of 3019) of the patients in our study population had a $10 \%$ or less, or low, predicted probability of CAD. Reassuringly, fewer than 5\% (14 of 301) of these patients actually had angiographic evidence of CAD, and only 1 patient-with left main coronary artery stenosis-had high-risk CAD (Table 2).

Of the 552 patients with diabetes or PVD, 55\% (304) had significant CAD compared with $29 \%$ (710) who were free of both these risk factors. Specifically, the absence of diabetes and PVD was associated with a $67 \%$ reduction in CAD risk relative to the presence of 1 or both variables (OR, 0.33; 95\% confidence interval, $0.27-0.4 ; P<.001)$. Moreover, and in agreement with our 4-variable model, 99.5\% (549 of 552) of patients in our study population with diabetes or PVD had a greater than $10 \%$ predicted probability of CAD.

We thus sought to identify gender-specific age thresholds associated with a $10 \%$ or less probability of 
TABLE 1. Baseline and perioperative characteristics of total cohort stratified by CCA status

\begin{tabular}{|c|c|c|c|c|}
\hline Variable & Total $(n=5463)$ & No $\operatorname{CCA}(n=752)$ & $\operatorname{CCA}(n=4711)$ & $P$ value* \\
\hline Mean age (y) & $65 \pm 15$ & $47 \pm 16$ & $68 \pm 13$ & $<.001$ \\
\hline Male gender & $3426(63)$ & $429(57)$ & $2997(64)$ & $<.001$ \\
\hline NYHA class III-IV & $3054(56)$ & $384(51)$ & $2670(57)$ & $<.001$ \\
\hline Angina & $1555(29)$ & $182(24)$ & $1373(29)$ & .005 \\
\hline Congestive heart failure & 787 (14) & $54(7)$ & $733(16)$ & $<.001$ \\
\hline Cerebrovascular accident & $213(4)$ & $11(1)$ & $202(4)$ & $<.001$ \\
\hline Diabetes mellitus & $886(16)$ & $44(6)$ & $842(18)$ & $<.001$ \\
\hline Hypercholesterolemia & $3879(71)$ & $395(53)$ & $3484(74)$ & $<.001$ \\
\hline Family history of CAD & $546(10)$ & $70(9)$ & $476(10)$ & .5 \\
\hline Hypertension & $3352(61)$ & $269(36)$ & $3083(65)$ & $<.001$ \\
\hline Previous MI & $489(9)$ & $18(2)$ & $471(10)$ & $<.001$ \\
\hline Obese (BMI $\left.\geq 30 \mathrm{~kg} / \mathrm{m}^{2}\right)$ & $1928(35)$ & $271(36)$ & $1657(35)$ & .65 \\
\hline Peripheral vascular disease & $362(7)$ & $16(2)$ & $346(7)$ & $<.001$ \\
\hline Smoker & $2847(52)$ & $327(43)$ & $2520(53)$ & $<.001$ \\
\hline Previous PCI & $500(9)$ & $23(3)$ & $477(10)$ & $<.001$ \\
\hline Ejection fraction $<50 \%$ & $664(12)$ & $38(5)$ & $626(13)$ & $<.001$ \\
\hline Surgical procedure & & & & $<.001$ \\
\hline Isolated aortic valve surgery & $2585(47)$ & $169(22)$ & $2416(51)$ & \\
\hline Isolated mitral valve surgery & $1551(28)$ & $236(31)$ & $1315(10)$ & \\
\hline Isolated septal myectomy & $762(14)$ & $277(37)$ & $485(10)$ & \\
\hline Combined procedure & $565(10)$ & $70(9)$ & $495(11)$ & \\
\hline Perioperative MI & $11(0.2)$ & $1(0.1)$ & $10(0.2)$ & .65 \\
\hline Operative mortality & $88(2)$ & $3(0.4)$ & $85(2)$ & .005 \\
\hline Hospital length of stay $\dagger$ (d) & $6(5-8)$ & $5(4-6)$ & $6(5-8)$ & $<.001$ \\
\hline
\end{tabular}

Data are presented as mean \pm standard deviation or $\mathrm{n}(\%)$, unless otherwise noted. CCA, Conventional coronary angiography; NYHA, New York Heart Association; $C A D$, coronary artery disease; $M I$, myocardial infarction; $B M I$, body mass index; $B S A$, body surface area; $P C I$, percutaneous coronary intervention. $* P$ value represents the comparison of patients with preoperative CCA versus those without CCA. †Data presented as median (interquartile range).

significant CAD to clarify which patients were at lowest risk. Overall, men aged 44 years or younger and women aged 52 years or younger had a low probability $(\leq 10 \%)$ of significant CAD. In the absence of diabetes and PVD, the age threshold was 45 years or younger and 55 years or younger for men and women, respectively
(Figure 2, A). Of the 2467 patients free of diabetes and PVD, 344 (14\%) were younger than the age thresholds consistent with a low probability of CAD at index surgery. Significant disease was detected in 16 of these 344 patients $(4.7 \%)$, only 1 of whom had high-risk CAD.

TABLE 2. Four-variable prediction models of significant CAD: overall and by surgical procedure

\begin{tabular}{|c|c|c|c|c|c|c|c|c|}
\hline \multirow[b]{2}{*}{ Category (n) } & \multirow[b]{2}{*}{ Variables } & \multirow[b]{2}{*}{ OR $(95 \% \mathrm{CI})$} & \multirow[b]{2}{*}{$P$ value } & \multirow[b]{2}{*}{ Model C-index* } & \multicolumn{4}{|c|}{ Predicted low probability ( $\leq \mathbf{1 0} \%$ probability of CAD) } \\
\hline & & & & & n $(\%)$ & Significant CAD $(\%)$ & LMA/3VD $(\%)$ & CABG $(\%)$ \\
\hline \multirow[t]{4}{*}{ All patients (3019) } & Age $\dagger$ & $1.4(1.3-1.5)$ & $<.001$ & 0.74 & $301 / 3019(10)$ & $14 / 301(4.7)$ & $1 / 301(0.3)$ & $8 / 301(3)$ \\
\hline & Male & $1.9(1.6-2.2)$ & $<.001$ & & & & & \\
\hline & DM & $2.3(1.8-2.8)$ & $<.001$ & & & & & \\
\hline & PVD & $2.7(1.9-4.0)$ & $<.001$ & & & & & \\
\hline \multirow[t]{4}{*}{ AVS (1668) } & Age $\dagger$ & $1.3(1.2-1.3)$ & $<.001$ & 0.69 & $17 / 1668(1)$ & $1 / 17(5.9)$ & $0 / 17(0)$ & $0 / 17(0)$ \\
\hline & Male & $1.9(1.5-2.4)$ & $<.001$ & & & & & \\
\hline & $\mathrm{DM}$ & $1.9(1.5-2.4)$ & $<.001$ & & & & & \\
\hline & PVD & $2.3(1.5-2.5)$ & $<.001$ & & & & & \\
\hline \multirow[t]{4}{*}{ MVS (1070) } & Age $\dagger$ & $1.5(1.3-1.5)$ & $<.001$ & 0.75 & $254 / 1070$ (24) & $12 / 254(4.7)$ & $2 / 254(0.7)$ & $8 / 254(3)$ \\
\hline & Male & $1.6(1.1-2.2)$ & .006 & & & & & \\
\hline & DM & $3.1(1.8-5.3)$ & $<.001$ & & & & & \\
\hline & PVD & $3.9(1.9-8.3)$ & $<.001$ & & & & & \\
\hline \multirow[t]{2}{*}{ SM (281) } & Age $\dagger$ & $1.3(1.2-1.7)$ & .001 & 0.70 & $148 / 281(53)$ & $8 / 148(5.4)$ & $0 / 148(0)$ & $5 / 148(3)$ \\
\hline & Male & $2.4(1.03-5.9)$ & .04 & & & & & \\
\hline
\end{tabular}

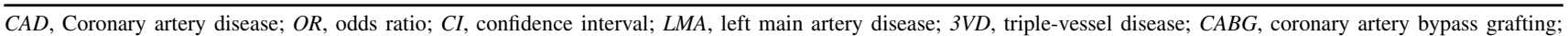
$D M$, diabetes mellitus; $P V D$, peripheral vascular disease; $A V S$, aortic valve surgery; $M V S$, mitral valve surgery; $S M$, septal myectomy. *After bootstrap validation, all adjusted $\mathrm{C}$-indexes were within 0.005 of the original value. $\dagger$ Age increase by 5 -y increments. 
All Patients

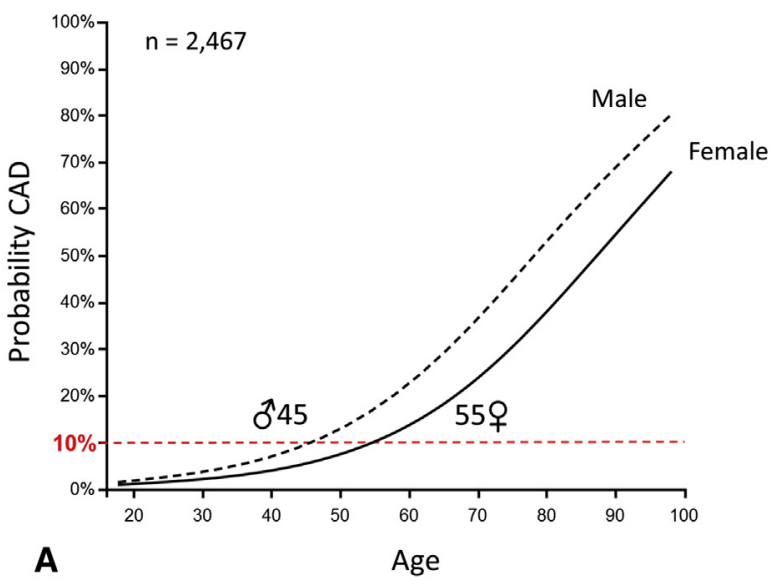

Mitral Valve Surgery

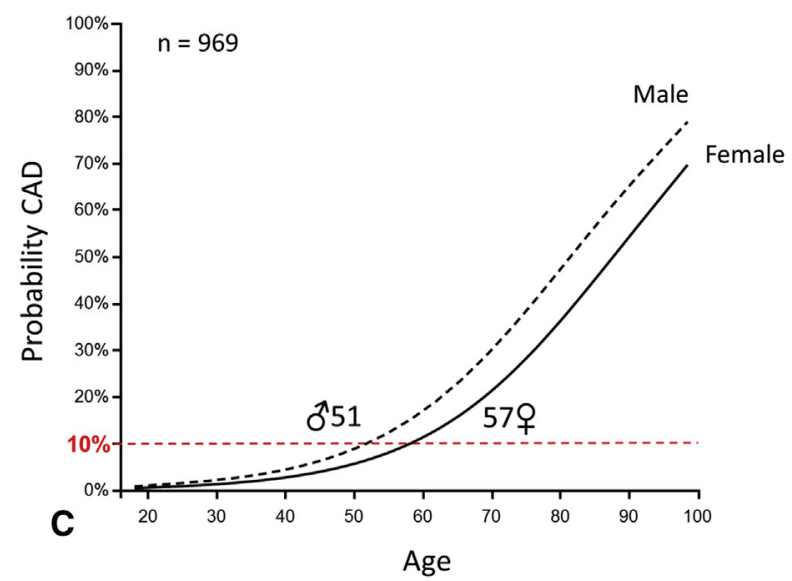

Aortic Valve Surgery

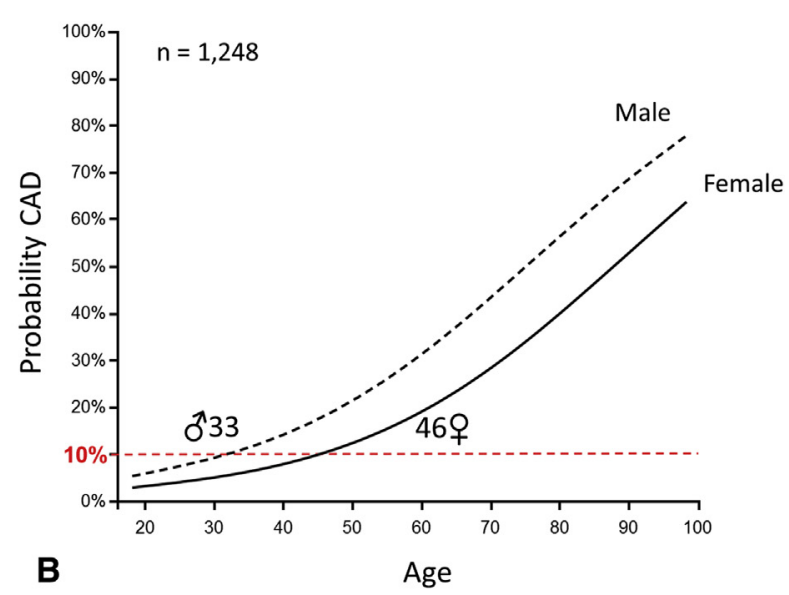

Septal Myectomy

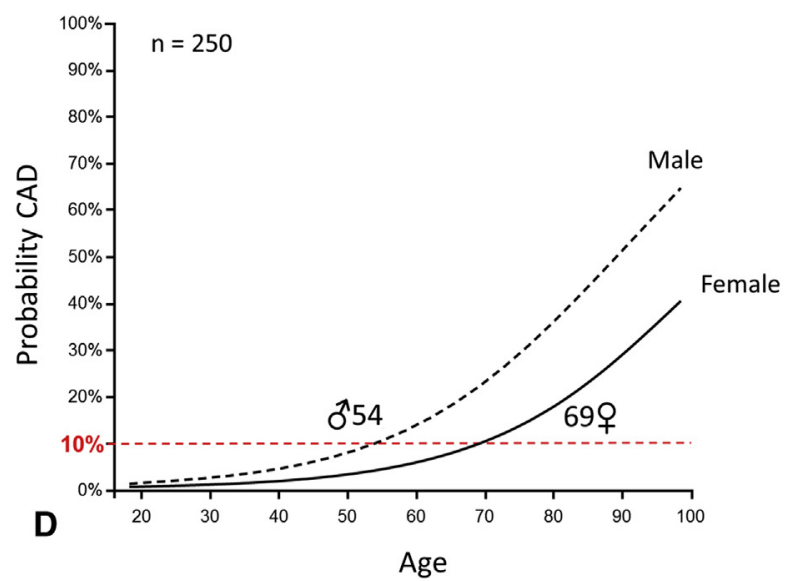

FIGURE 2. Gender-specific age thresholds predicting low probability of coronary artery disease $(C A D)$ in the absence of diabetes and peripheral vascular disease $(P V D)$. A, Age thresholds consistent with a $10 \%$ or less predicted probability of CAD in all men and women free of diabetes and PVD undergoing noncoronary cardiac surgery. Gender-specific age thresholds are also depicted for subsets of patients undergoing (B), aortic valve surgery, (C), mitral valve surgery, and (D), septal myectomy.

\section{CAD Prediction by Surgical Subset}

We further sought to generate separate risk prediction models and to establish age-threshold "rules" for the subsets of patients who had undergone AVS, MVS, or SM. AVS group. AVS was performed in 1668 of 3019 (55\%) patients, of whom $1465(88 \%)$ had predominant aortic valve stenosis. Of the 1668 patients, 1611 (97\%) underwent aortic valve replacement. Significant coronary stenosis was present on CCA in $44 \%$ (726 of 1668) of patients, and concomitant CABG was performed in 33\% (549 of 1668) of patients. The most predictive model for significant CAD again included older age, male gender, diabetes, and PVD (C-index $=0.69$; Table 2 ). The age cutoff consistent with a low probability of significant CAD at AVS was 31 years or younger in men and 44 years or younger in women. In the absence of diabetes and PVD, the age threshold increased slightly to 33 years or younger and 46 years or younger in men and women, respectively (Figure 2,B).

MVS group. Of the 1070 patients who underwent MVS, moderate or severe valve regurgitation was evident in 1035 (97\%), and valve repair was performed in 905 $(85 \%)$, including those with regurgitation, stenosis, and leaflet tethering or calcification. CCA detected CAD in one fourth (257 of 1070) of patients, and concomitant CABG was performed in 17\% (180 of 1070). In the MVS population, older age, male gender, diabetes, and PVD remained the most prominent predictors of $\mathrm{CAD}$ $(\mathrm{C}$-index $=0.75$; Table 2$)$. Age 50 years or younger in men and 54 years or younger in women predicted a $10 \%$ or less probability of significant CAD. The age threshold increased slightly to 51 and 57 years in men and women free of diabetes and PVD, respectively (Figure 2, C). 
SM group. Significant CAD was diagnosed in 31 of 281 $(11 \%)$ of those in the SM cohort, and concomitant CABG was performed in $19(7 \%)$. Because of the small number of events in the SM subset $(n=31)$, the use of 4 variables in a predictive model would have resulted in overfitting and was hence inappropriate. Although the top 2 predictors remained age and male gender, the addition of a third variable contributed minimally to overall model discrimination. Thus, among patients undergoing SM, we opted to fit a 2-variable model, with older age and male gender (C-index $=0.70$; Table 2). Of all 281 patients undergoing $\mathrm{SM}$, a $10 \%$ or less probability of CAD was predicted for men aged 52 years or younger and for women aged 66 years or younger. In the absence of diabetes and PVD, the age threshold increased to 54 and 69 years for men and women, respectively (Figure 2,D).

\section{DISCUSSION}

Although the practice of preoperative CCA in patients undergoing noncoronary cardiac surgery has become almost universal, the diagnostic yield of CCA in contemporary practice is unclear. We sought to characterize the burden of significant CAD among patients undergoing noncoronary cardiac surgery and to establish simple bedside models predicting the probability of coexistent coronary stenosis in the absence of classic disease risk factors. We detail herein that preoperative CCA reveals significant coronary stenosis in less than $50 \%$ of patients undergoing noncoronary cardiac surgery and in only one third of those without angina, previous MI, or PCI. As such, we constructed risk prediction models and identified gender-specific age thresholds that successfully detected patients with a low probability of CAD. These preliminary findings support the notion that a great number of cardiac surgical patients are exposed to this invasive and expensive procedure than is necessary. ${ }^{5,6}$

An important argument favoring routine CCA before cardiac surgery is that severe coexistent CAD, left untreated at noncoronary cardiac surgery, can lead to catastrophic short(and long-) term outcomes. ${ }^{1,2}$ Additionally, the cost and morbidity associated with the need for postoperative PCI or repeat sternotomy for CABG is not insignificant. The addition of concomitant CABG when indicated at index surgery has thus proved efficacious and safe. ${ }^{1,2,24}$ The widespread performance of preoperative CCA has been supported by current surgical guidelines recommending angiography before valve surgery for all men aged 35 years or older, premenopausal women aged 35 years or older with cardiac risk factors, and postmenopausal women. ${ }^{4}$ Limited guidance is available for patients undergoing surgical myectomy; however, special concerns may exist for this population, including the need for concomitant coronary unroofing to treat myocardial bridging. ${ }^{25,26}$ These and other factors have spawned the liberalized performance of preoperative CCA before noncoronary cardiac surgery, which in our population was performed in approximately $90 \%$ of patients.

Despite a high sensitivity for the detection of CAD, guideline specificity has been estimated to be as low as $1 \%$ to $3 \%,{ }^{5,6}$ raising questions regarding the diagnostic yield of this strategy. Evaluating almost 400,000 patients undergoing elective CCA, Patel et $\mathrm{al}^{9}$ recently reported that significant coronary stenosis was evident in only one third of angiograms. Importantly, their analysis excluded patients undergoing valvular surgery, who account for most subjects undergoing noncoronary surgery, ${ }^{27}$ and in subsets of whom, the risk of underlying CAD may be increased. ${ }^{10}$ In our large, contemporary series investigating the practice of CCA among cardiac surgical candidates, we found that $45 \%$ of the 4711 patients who underwent CCA, and two thirds of those with angina, previous MI, or PCI, had significant CAD. This is unsurprising, given that MI and PCI are markers of previous coronary flow limitation. Moreover, our findings are consistent with historic reports estimating that up to one half of patients with valve pathology and symptoms of angina have underlying CAD. ${ }^{28}$ Therefore, our findings support a strategy of CCA for patients with angina, previous MI, or PCI at the time of index surgery.

Conversely, significant CAD was only observed in one third of the 3019 patients free of angina, previous MI, or PCI. Leveraging this cohort, we successfully constructed and internally validated 4-variable models with very good discriminative ability (C-index, 0.69-0.75). Specifically, the overall model identified that $10 \%$ of patients currently undergoing CCA had a $10 \%$ or less probability of CAD. Subset models (Table 2) were able to identify that approximately $25 \%$ of patients undergoing MVS and more than $50 \%$ of those undergoing SM were at low risk of CAD. We found slightly different results in the patients undergoing AVS—of whom, only $1 \%$ had a low probability of $\mathrm{CAD}$, underlining the increased prevalence of coronary stenosis in patients undergoing AVS, likely related to the common risk factors associated with the development of coronary atherosclerosis and senile calcific aortic valve disease. ${ }^{11-13}$ Reassuringly, in all models, only $4 \%$ to $6 \%$ of patients classified as having a $10 \%$ or less probability of CAD had significant coronary stenosis, and less than $1 \%$ had high-risk disease, supporting the potential utility of these models.

Our present report examined all patients who had undergone AVS, MVS, and SM, thus expanding generalizability of our findings among the large subpopulations of patients undergoing preoperative workup for noncoronary cardiac surgery. Previous prediction algorithms for the detection of significant CAD were developed in specific surgical subsets, including patients with degenerative mitral valve 


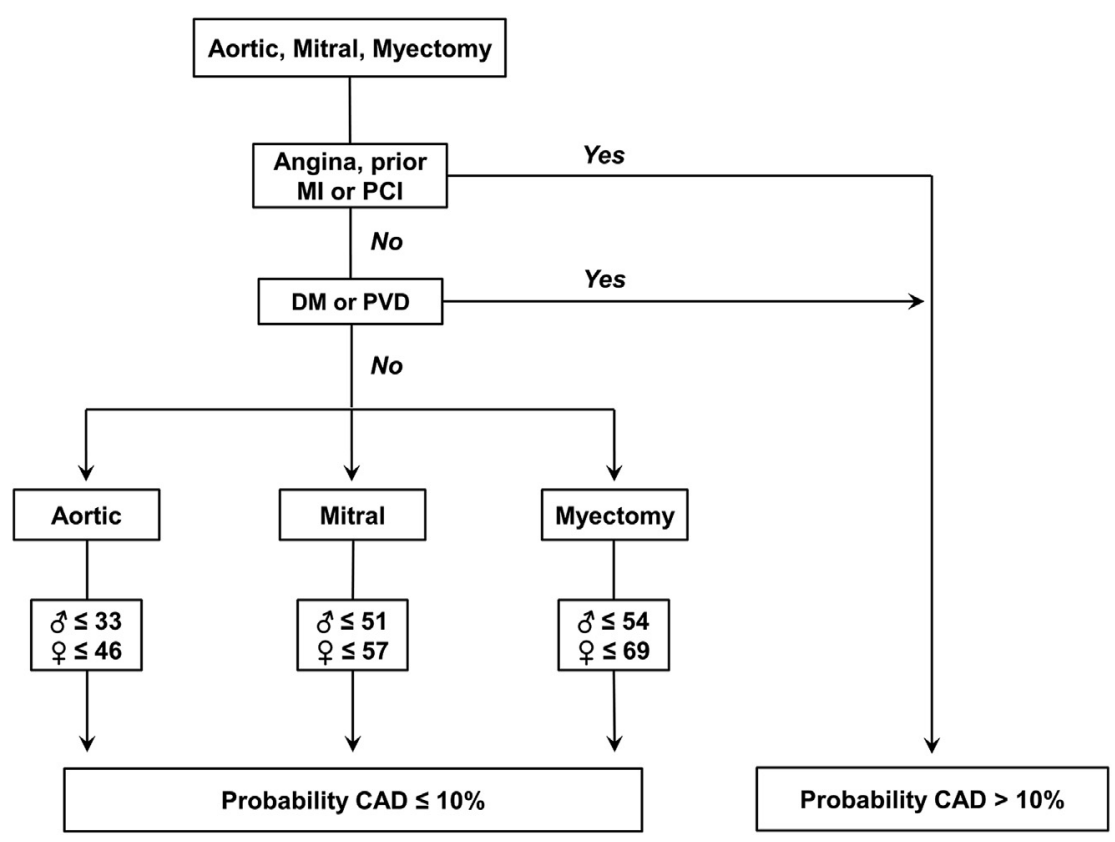

FIGURE 3. Algorithm for estimating probability of coronary artery disease prior to noncoronary cardiac surgery. MI, Myocardial infarction;

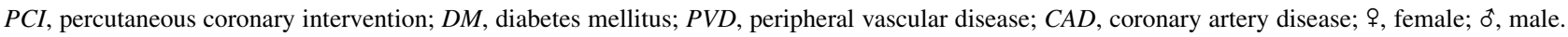

prolapse $^{5,6}$ and rheumatic valve disease. ${ }^{29,30}$ However, lengthy calculations required for these logistic regression models ${ }^{6}$ have likely limited their bedside utility and widespread clinical adoption. Lim et al, ${ }^{5}$ among others, have attempted to circumvent the need for complex calculations by developing additive scoring systems to predict CAD. However, to our knowledge, these models have not been incorporated into routine patient care. We are attempting to address these limitations through the development of a Smartphone application and online medical calculators to reproduce our risk models in expanded form.

The present study has established straightforward principles that are easily applicable at the patient bedside and provides practitioners with an enhanced ability to adjudicate patient CAD risk beyond the current broad-based and highly generalized guidelines. ${ }^{4}$ Specifically, older age, male gender, diabetes, and PVD were consistently the most robust predictors of CAD. Given that more than one half of the patients with diabetes or PVD had significant CAD, and more than $99 \%$ of patients with either of these risk factors had a greater than $10 \%$ predicted probability of coronary stenosis, our data indicate that patients with diabetes or PVD are unlikely to be at low risk of coronary stenosis and, as a general rule, may be best served by preoperative CCA. In contrast, in those free of diabetes and PVD at the time of preoperative assessment for index surgery, we identified gender-specific age thresholds consistent with a $10 \%$ or less probability of CAD, for whom preoperative CCA could potentially be avoided
(Figure 3). The guidance is not exhaustive and cannot conceivably be expected to completely rule out the existence of CAD. Our findings are thus not intended to be taken as substitutes for sound clinical judgment nor the abandonment of traditional risk stratification schemes. Future validation of these findings in large multicenter studies will be important. Furthermore, the question of "if not CCA, then what?" can be justifiably posed. Computed tomography coronary angiography has emerged as a promising and efficacious modality by which coronary obstructions can be identified. ${ }^{31,32}$ Although not yet approved for routine evaluation of coronary anatomy before cardiac surgery, it has been proposed that instead of replacing CCA, computed tomography coronary angiography may assume the role of a "gatekeeper": differentiating between patients free of CAD and those requiring additional investigation using CCA. ${ }^{33}$ Additional studies detailing the cost, efficacy, and safety ratios of various diagnostic tests are necessary, and are beyond the scope of the present report.

Importantly, recent trials have demonstrated no advantage for revascularization versus medical therapy in stable patients with less extensive but "significant" $\mathrm{CAD}^{7,8}$ It could thus be proposed that the goal of preoperative evaluation should perhaps be to identify patients with left main, multivessel, or critical left anterior descending artery disease to undergo CABG. Meanwhile, patients with less severe CAD may be best served by conservative coronary management at the time of index surgery. 


\section{Study Limitations}

Although we investigated a broad range of patients undergoing noncoronary cardiac surgery, the generalizability of our prediction models remains limited to those undergoing AVS, MVS, or SM, and model applicability in other surgical subsets remains to be determined. Thus, despite internal model validation, future confirmation in external populations is recommended as a bridge toward widespread dissemination into clinical practice. Importantly, we defined a $10 \%$ or less risk as being consistent with a low probability of CAD. Although a relatively subjective threshold that could be challenged as arbitrary, this decision was guided by previous data ${ }^{18-22}$ and also by the consensus judgment of expert cardiologists and cardiovascular surgeons at our institution. Finally, although the proposed models do not detect all patients free of CAD at CCA, they do nevertheless identify a substantial proportion of patients currently subject to an invasive and costly procedure who might at some time in the future be candidates for other less-invasive and more affordable screening modalities. Thus, when considered in the context of the current widespread practice of CCA, our models represent an important step toward optimizing efficiency of resource allocation while maintaining a high quality of patient care.

\section{CONCLUSIONS}

Almost $90 \%$ of subjects undergoing noncoronary cardiac surgery undergo preoperative CCA. However, in the absence of angina, previous MI, or PCI, CCA identifies significant $\mathrm{CAD}$ in only one third of patients. Clinical prediction models can facilitate the identification of subjects at low risk of CAD, in whom preoperative CCA might potentially be avoided or substituted prior to noncoronary cardiac surgery. Ultimately, this strategy may minimize procedure-related morbidity, decrease cost, and improve value in health care delivery.

\section{References}

1. Lund O, Nielsen T, Pilegaard H, Magnussen K, Knudsen M. The influence of coronary artery disease and bypass grafting on early and late survival after valve replacement for aortic stenosis. J Thorac Cardiovasc Surg. 1990;100: 327-37.

2. Mullany CJ, Elveback LR, Frye RL, Pluth JR, Edwards WD, Orszulak TA, et al. Coronary artery disease and its management: influence on survival in patients undergoing aortic valve replacement. J Am Coll Cardiol. 1987;10:66-72.

3. Morrison G, Thomas R, Grimmer S, Silverton P, Smith D. Incidence of coronary artery disease in patients with valvular heart disease. Br Heart J. 1980;44:630-7.

4. Bonow RO, Carabello BA, Chatterjee K, De Leon AC, Faxon DP, Freed MD, et al. ACC/AHA 2006 guidelines for the management of patients with valvular heart disease: a report of the American College of Cardiology/American Heart Association Task Force on Practice Guidelines (Writing Committee to Develop Guidelines for the Management of Valvular Heart Disease). J Am Coll Cardiol. 2006;48:e1-148.

5. Lim E, Ali ZA, Barlow CW, Jackson CH, Halstead JC, Barlow JB, et al. A simple model to predict coronary disease in patients undergoing operation for mitral regurgitation. Ann Thorac Surg. 2003;75:1820-5.

6. Lin SS, Lauer MS, Asher CR, Cosgrove DM, Blackstone E, Thomas JD, et al. Prediction of coronary artery disease in patients undergoing opera- tions for mitral valve degeneration. J Thorac Cardiovasc Surg. 2001;121: 894-901.

7. Boden WE, O'Rourke RA, Teo KK, Hartigan PM, Maron DJ, Kostuk WJ, et al. Optimal medical therapy with or without PCI for stable coronary disease. $N$ Engl J Med. 2007;356:1503-16.

8. Frye RL, August P, Hardison RM, Kelsey SF, MacGregor JM, Orchard TJ, et al. A randomized trial of therapies for type 2 diabetes and coronary artery disease. N Engl J Med. 2009;360:2503-15.

9. Patel MR, Peterson ED, Dai D, Brennan JM, Redberg RF, Anderson HV, et al. Low diagnostic yield of elective coronary angiography. N Engl J Med. 2010; 362:886-95.

10. Chandra HR, Goldstein JA, Choudhary N, O'Neill CS, George PB, Gangasani SR, et al. Adverse outcome in aortic sclerosis is associated with coronary artery disease and inflammation. J Am Coll Cardiol. 2004;43:169-75.

11. Peltier M, Trojette F, Sarano ME, Grigioni F, Slama MA, Tribouilloy CM. Relation between cardiovascular risk factors and nonrheumatic severe calcific aortic stenosis among patients with a three-cuspid aortic valve. Am J Cardiol. 2003;91:97-9.

12. Stewart BF, Siscovick D, Lind BK, Gardin JM, Gottdiener JS, Smith VE, et al. Clinical factors associated with calcific aortic valve disease. J Am Coll Cardiol. 1997;29:630-4.

13. Miller JD, Weiss RM, Heistad DD. Calcific aortic valve stenosis: methods, models, and mechanisms. Circ Res. 2012;108:1392-412.

14. Society of Thoracic Surgeons Adult Cardiac Surgery Data Specifications. Available from http://www.sts.org/sts-national-database/database-managers/ adult-cardiac-surgery-database/data-collection. Accessed April 14, 2013.

15. Scanlon PJ, Faxon DP, Audet A, Carabello B, Gregory J, Eagle KA, et al. ACC/ AHA guidelines for coronary angiography: report of the American College of Cardiology/American Heart Association Task Force on Practice Guidelines (Committee on Coronary Angiography). J Am Coll Cardiol. 1999;33:1756-824.

16. Breiman L. Random forests. Machine Learning. 2001;45:5-32.

17. Harrell F. Regression Modeling Strategies: With Applications to Linear Models, Logistic Regression, and Survival Analysis. Springer Series in Statistics. New York: Springer; 2001.

18. Hennessy TG, Codd MB, Kane G, McCarthy C, McCann HA, Sugrue DD. Dobutamine stress echocardiography in the detection of coronary artery disease: importance of the pretest likelihood of disease. Am Heart J. 1997;134:685-92.

19. Gibbons RJ, Lee KL, Pryor D, Harrell FE, Coleman RE, Cobb FR, et al. The use of radionuclide angiography in the diagnosis of coronary artery disease- $\mathrm{a}$ logistic regression analysis. Circulation. 1983;68:740-6.

20. Melin JA, Wijns W, Vanbutsele RJ, Robert A, De Coster P, Brasseur LA, et al. Alternative diagnostic strategies for coronary artery disease in women: demonstration of the usefulness and efficiency of probability analysis. Circulation. 1985;71:535-42.

21. Gibbons RJ, Balady GJ, Beasley JW, Bricker JT, Duvernoy WFC, Froelicher VF, et al. ACC/AHA guidelines for exercise testing: a report of the American College of Cardiology/American Heart Association Task Force on Practice Guidelines (Committee on Exercise Testing. J Am Coll Cardiol. 1997;30:260-315.

22. Diamond GA, Forrester JS, Hirsch M, Staniloff HM, Vas R, Berman DS, et al. Application of conditional probability analysis to the clinical diagnosis of coronary artery disease. J Clin Invest. 1980;65:1210-21.

23. Liaw A, Wiener M. Classification and regression by randomForest. $R$ News. 2002; $2: 18-22$.

24. Czer L, Gray R, Stewart M, De Robertis M, Chaux A, Matloff J. Reduction in sudden late death by concomitant revascularization with aortic valve replacement. J Thorac Cardiovasc Surg. 1988;95:390-401.

25. Gersh BJ, Maron BJ, Bonow RO, Dearani JA, Fifer MA, Link MS, et al. 2011 ACCF/AHA guideline for the diagnosis and treatment of hypertrophic cardiomyopathy: a report of the American College of Cardiology Foundation/American Heart Association Task Force on Practice Guidelines. Circulation. 2011;124: e783-831.

26. Alegria JR, Herrmann J, Holmes DR, Lerman A, Rihal CS. Myocardial bridging. Eur Heart J. 2005;26:1159-68.

27. Society of Thoracic Surgeons Adult Cardiac Surgery Database Executive Summary. Available at: http://www.sts.org/sts-national-database/databasemanagers/executive-summaries. Accessed February 23, 2013.

28. Exadactylos N, Sugrue DD, Oakley CM. Prevalence of coronary artery disease in patients with isolated aortic valve stenosis. Br Heart J. 1984;51:121-4.

29. Li SC, Liao XW, Li L, Zhang LM, Xu ZY. Prediction of significant coronary artery disease in patients undergoing operations for rheumatic mitral valve disease. Eur J Cardiothorac Surg. 2012;41:82-6. 
30. Yan T, Zhang G, Li B, Han L, Zang J, Li L, et al. Prediction of coronary artery disease in patients undergoing operations for rheumatic aortic valve disease. Clin Cardiol. 2012;35:707-11.

31. Miller JM, Rochitte CE, Dewey M, Arbab-Zadeh A, Niinuma H, Gottlieb I, et al. Diagnostic performance of coronary angiography by 64-row CT. $N$ Engl J Med. 2008;359:2324-36.

32. Mollet NR, Cademartiri F, Van Mieghem CAG, Runza G, McFadden EP, Baks T, et al. High-resolution spiral computed tomography coronary angiography in patients referred for diagnostic conventional coronary angiography. Circulation. 2005; 112:2318-23.

33. Meijboom WB, Mollet NR, Van Mieghem CAG, Kluin J, Weustink AC, Pugliese F, et al. Pre-operative computed tomography coronary angiography to detect significant coronary artery disease in patients referred for cardiac valve surgery. J Am Coll Cardiol. 2006;48:1658-65.

\section{Discussion}

Dr Jennifer Sue Lawton (St Louis, Mo). Congratulations on very nice, very useful work and a very nice presentation. I really enjoyed your algorithm and the specificity for the different types of valve pathology. As you know, we do not know the incidence of significant CAD in the general population, and the study you quoted from the New England Journal of Medicine by Patel et al-they did not include valve surgery patients, and they used different criteria for significant CAD. Your criterion was greater than $50 \%$ stenosis in an epicardial vessel and theirs was $70 \%$ or $50 \%$ in the left main. They reported on 400,000 patients, approximately, about one third of whom were asymptomatic but had disease, but for some reason those patients had undergone cardiac catheterization. Similarly, in the 2008 updates of the American Heart Association guidelines, they state that in an asymptomatic population, the incidence is approximately $4 \%$. So, with that in mind, looking at your stratification of your study population, 752 patients were ruled out fairly soon if they had not undergone preoperative catheterization within 6 months of the valve surgery. Thus, for those patients, I have a question, if you have the data, about them, because in your report you did mention that a few of them had had MI or PCI before valve surgery. So, did they not undergo cardiac catheterization before surgery by surgeon preference or were they young patients with mitral regurgitation? If you have any data on those patients, because, with that in mind, your estimation of risk and your conclusions might have been underestimated or overestimated. That is my first question.

Dr Thalji. Thank you very much for your comments, Dr Lawton, and for your very insightful question. You raise an important point. Specifically, that a subset of patients that was excluded, owing to the absence of preoperative angiography within 6 months of surgery, did indeed have symptoms of angina, previous PCI, or previous MI. There are several potential explanations for this.

First, we defined preoperative angiography as being within 6 months of the index surgery. Some patients had undergone preoperative angiography that was performed beyond this period; for instance, within 6 months to 1 year. However, after consultation with the cardiologists and cardiac surgeons, angiography performed more than 6 months before surgery were deemed less likely to be representative of the baseline burden of CAD.

Furthermore, it is also worthwhile noting that about $20 \%$ of the patients who did not undergo conventional invasive angiography did alternatively undergo computed tomography coronary angiography. Most of these patients were those undergoing robotic mitral valve repair, which, at our institution, is performed by Drs Rakesh
Suri and Harold Burkhart. It is standard practice at our institution for all patients who undergo robotic cardiac surgery to undergo computed tomography of the chest, abdomen, and pelvis to assess for underlying vascular disease, including coronary stenosis. It is, therefore, possible that these patients who had had MI, PCI, or angina had been assessed by a computed tomographic angiogram, which proved to be negative, hence circumventing the need for conventional angiography.

Dr Lawton. Do you have data for those 752 patients and whether any of them did undergo concomitant CABG with valve surgery?

Dr Thalji. That is a good question. Yes, that is correct. Approximately 25 patients without angiography within 6 months of surgery did require concomitant $\mathrm{CABG}$. The situation for these patients, as before, was such that the surgeons believed that CCA more than 6 months before surgery was sufficient to guide surgical practice and perform concomitant $\mathrm{CABG}$. This highlights that our algorithms are simply guides and do not outweigh the sound clinical judgment of the surgeon.

Dr Lawton. My next question is, sort of as a devil's advocate, a number of surgeons are cautious and very conservative; thus, how would you convince such surgeons to forego cardiac catheterization preoperatively when we know additional data can be gained from the study? It often provides a very nice look at the ascending aorta and an assessment of left ventricular function. Additional findings could be that that the patient has anomalous coronary anatomy, such as a double-barrel left main, which could become important, depending on your cardioplegia strategy. Similarly, if the patient is right dominant and you plan a very long mitral repair, perhaps that will not work and requires a replacement and you have only given retrograde cardioplegia. A number of scenarios are possible, so how could you convince us we do not need that information?

Dr Thalji. That is a great question, and there are several points to be made. First, with the pending approval of the Affordable Care Act, we will all be called on to scrutinize our actions and assess the associated costs. Our current investigation is the first step of multiple studies that are forthcoming to determine whether we are overusing coronary angiography. However, are we suggesting that surgeons should abandon angiography altogether? No, we do not believe this should be the case. What we are suggesting is that perhaps subsets of patients who specifically are at a low risk of CAD might be able to either forego invasive angiography or, alternatively, undergo other less-invasive investigative procedures to evaluate coronary obstruction. For instance, ventricular function can potentially be assessed using echocardiography. To delineate the coronary anatomy, computed tomography coronary angiography has become a very popular topic examined in contemporary studies. I think it is going to take a bit of time before we see a marked shift in investigative habits; however, we need to start being self-critical of our clinical practice and to evaluate the effect it has on patient safety and on healthcare economics.

Dr David C. McGiffin (Birmingham, Ala). Has there been any change at the clinic since this information, and if so, what do you do now?

Dr Thalji. To date, what we can say is that there has been a change in our mind sets. Specifically, we have come to appreciate that a large divide exists between what we are currently doing and perhaps what we should be doing. Importantly, validation of our models in external populations is a critical and necessary step 
that needs to be undertaken before we should drastically alter our clinical practice.

Dr McGiffin. So it has not translated into a change yet?

Dr Thalji. To date, it has not.

Dr Shyam Kolvekar (London, United Kingdom). I enjoyed your report, and I think your risks come with these problems. I have 2 small questions. One, did you prefer stress echocardiography compared to computed tomography angiography to find the patients who do not have a high risk and to determine whether they have any obstructive disease, because it is less invasive? The second question is, did you have any complications with your angiography patients where they had morbidity or mortality?

Dr Thalji. Thank you for your questions. Regarding your first question, you are correct, the published data have shown that stress echocardiography can be leveraged to obtain valuable information regarding the coronary disease burden. Although stress echocardiography is a less-invasive option, as a general rule of thumb, clinicians have tended to have a greater peace of mind when the coronary anatomy has been visualized, such as is the case with angiography. These are certainly factors that merit consideration when determining which preoperative investigations to perform.

Regarding your second question, in terms of complications, we do not have that data available for our population at the moment.
However, we can allude to previously published data detailing that the rate of major complications at invasive angiography is approximately $2 \%$. However, as I said, we do not have that data for our patients specifically.

Dr Rakesh M. Suri (Rochester, Minn). Just to comment on Nassir's very thoughtful answer and to respond to 1 of the last questions. This is a large, retrospective population-based study, and it was not designed to answer the specific question-if coronary angiography is avoided, which surrogate tests or which replacement tests should be ordered instead - that is a topic that will be addressed in forthcoming investigations. We will be better prepared to answer that as we move forward.

Dr A. Pieter Kappetein (Rotterdam, The Netherlands). Did you consider the type of valvular heart disease, whether that made a difference, whether it was stenotic, aortic valve disease, or insufficiency?

Dr Thalji. That is a good question. We have not presented such data. Nevertheless, in a subset analysis, we found that aortic stenosis was an important variable predictive of underlying significant CAD. Similarly, and as we have documented in our study, patients undergoing AVS - the vast majority of whom had aortic stenosis-(1) were more likely to have coexistent $\mathrm{CAD}$, and (2) were found to have coronary stenosis at a much younger age compared with those undergoing MVS or SM. 
TABLE E1. Predictors of significant CAD in patients without angina or previous MI or PCI $(n=3019)$

\begin{tabular}{|c|c|c|c|c|c|}
\hline \multirow[b]{2}{*}{ Variable } & \multirow[b]{2}{*}{ Patients (n) } & \multicolumn{2}{|c|}{ Univariate Analysis } & \multicolumn{2}{|c|}{ Multivariate Analysis $\dagger$} \\
\hline & & OR $(95 \%$ CI $)$ & $P$ value & OR $(95 \%$ CI $)$ & $P$ value \\
\hline Age (per 5 y increment)* & - & $1.40(1.35-1.45)$ & $<.001$ & $1.40(1.34-1.47)$ & $<.001$ \\
\hline Male gender & $1910(63)$ & $1.42(1.21-1.66)$ & $<.001$ & $1.85(1.54-2.23)$ & $<.001$ \\
\hline Smoking history & $1528(51)$ & $1.52(1.30-1.77)$ & $<.001$ & $1.30(1.10-1.55)$ & .003 \\
\hline $\mathrm{DM}$ & $442(15)$ & $2.68(2.18-3.29)$ & $<.001$ & $2.01(1.61-2.53)$ & $<.001$ \\
\hline Hypertension & $1821(60)$ & $2.09(1.78-2.46)$ & $<.001$ & $1.36(1.13-1.63)$ & $<.001$ \\
\hline Hypercholesterolemia & $2083(69)$ & $1.77(1.49-2.10)$ & $<.001$ & $1.49(1.23-1.80)$ & $<.001$ \\
\hline Family history CAD & $250(8)$ & $1.68(1.29-2.18)$ & $<.001$ & $1.98(1.47-2.65)$ & $<.001$ \\
\hline PVD & $158(5)$ & $4.01(2.86-5.61)$ & $<.001$ & $2.49(1.72-3.62)$ & $<.001$ \\
\hline NYHA class III-IV & $1395(46)$ & $1.38(1.19-1.61)$ & $<.001$ & - & - \\
\hline Obese $\left(\mathrm{BMI} \geq 30 \mathrm{~kg} / \mathrm{m}^{2}\right)$ & $985(33)$ & $0.94(0.80-1.11)$ & .47 & - & - \\
\hline $\mathrm{EF}<50 \%$ & $310(10)$ & $1.56(1.25-1.98)$ & $<.001$ & - & - \\
\hline CVA & $116(4)$ & $2.11(1.46-3.06)$ & $<.001$ & - & - \\
\hline
\end{tabular}

